\title{
Expertise
}

\section{La 5ème génération de réseaux mobiles: Des données accessibles à très haute vitesse}

\section{Expertise}

\section{The 5th generation of mobile networks: Accessible data at very high}

\section{speeds}

\author{
Jean-Louis Monino ${ }^{1}$ \\ ${ }^{1}$ Montpellier Recherche en Economie - Université de Montpellier, jean-louis.monino@umontpellier.fr
}

RÉSUMÉ. Dans un environnement en constante évolution, les opérateurs de télécommunications ont besoin des meilleures technologies pour répondre à leurs besoins commerciaux. La nouvelle génération va améliorer et créer de nouvelles utilisations qui ne peuvent pas être satisfait par les technologies actuelles. La latence, le débit et la fiabilité entre autres, sont essentielles pour répondre aux nouvelles notions d'utilisation et de fabrication.

L'évolution continue des réseaux va au-delà des générations précédentes de communication mobile. Les capacités comprennent une capacité système massive, des débits de données très élevés partout, une latence très faible, une fiabilité et une disponibilité très élevées, un coût et une consommation d'énergie beaucoup plus faibles. Le tranchage de réseau va permettre aux opérateurs de fournir des réseaux virtuels dédiés avec des fonctionnalités spécifiques au client, le Distributed Cloud va permettre de placer les charges de travail plus près de la périphérie pour une meilleure qualité de service telle que la latence, un apprentissage automatique en temps réel et intelligence artificielle (IA), l'analyse sera importante pour permettre l'auto-optimisation des réseaux et les sécuriser. Dans les télécommunications, un accord de niveau de service (SLA) définit un ensemble de services spécifiques adaptés aux besoins de chacun des clients. Les opérateurs pourront profiter des avantages de la 5G pour satisfaire leurs clients et favoriser les différentes chaînes de valeur de la $5 \mathrm{G}$ pour développer la future entreprise.

ABSTRACT. In an ever-changing environment, telecom operators need the best technology to meet their business needs. The new generation will improve and create new uses that cannot be satisfied by current technologies. Latency, throughput and reliability, among others, are essential to meet new usage and manufacturing concepts.

The continuous evolution of networks goes beyond previous generations of mobile communication. Capabilities include massive system capacity, very high data rates, reliability and availability, very low latency, and much lower cost and power consumption. Network slicing will allow operators to provide dedicated virtual networks with customer-specific features. Distributed Cloud will allow workloads to be placed closer to the edge for better quality of service such as latency, real-time machine learning and artificial intelligence (Al). Analysis will be important in enabling self-optimization and network security.

In telecommunications, a Service Level Agreement (SLA) defines a set of specific services adapted to the needs of each customer. Operators will be able to take advantage of the benefits of $5 \mathrm{G}$ to satisfy their customers and promote the different $5 \mathrm{G}$ value chains in order to develop the future business.

MOTS-CLÉS. 5G, analogique, numérique, internet mobile, usine 4.0, industrie 4.0.

KEYWORDS. 5G, analog, digital, mobile internet, factory 4.0, industry 4.0.

\section{Introduction}

La 5G, elle frappe déjà à notre porte, avec à la clé des débits dépassant les $10 \mathrm{Gbit} / \mathrm{s}$ ! « La 5G a un problème de nom, expliquait Thierry Boisnon, le patron de Nokia France. Ce n'est pas une simple évolution de la $4 \mathrm{G} \gg$ C'est en réalité une véritable technologie de rupture qui ouvrira des perspectives de développement des usages numériques. Elle pourrait ainsi bénéficier à la plupart de nos objets connectés, de la voiture autonome à la télémédecine, ...

Pour Jacques Attali, économiste et Président de Positive Planet, la 5G apportera « une amélioration massive de la vitesse de collecte et de traitement des données, ... la 5G a d'autres objectifs : elle permettra en effet d'abord de déployer les moyens d'une chirurgie de précision à distance et de faire 
fonctionner des voitures autonomes, ce qui nécessite l'un et l'autre des temps de réaction extrêmement brefs »

Pour SFR «C'est un tout nouveau réseau mobile avec un débit internet jusqu'à 10 fois plus rapide et plus performant que la $4 \mathrm{G}$ » c'est la promesse d'un débit qui sera décuplé, mais surtout le réseau sera extrêmement réactif. Les temps de latence, à savoir les délais de transit d'une donnée entre le moment où elle est envoyée et celui où elle est reçue, seront réduits à 1 milliseconde, ainsi nous pourrons avoir des accès de transfert de données quasi-simultané, ... avec une vitesse de connexion 100 fois plus rapide que la 4G, atteignant jusqu'à $100 \mathrm{Gbit} / \mathrm{s}$ et une très faible latence. Ainsi, la 5G doit-être capable de supporter une multitude d'objets connectés simultanément, sans risque de saturation. Cette connexion accrue des objets aura pour but final de générer une profonde évolution de nos modes de vie traditionnels au travers de nouvelles innovations. Cette révolution technologique pose la question de la maitrise et de la gestion de l'intégration de l'ensemble de ces objets connectés en mobilité que ce soit dans un cadre de vie privé, ou dans un cadre de vie professionnel.

L'arrivée de la $5 \mathrm{G}$ est bien plus médiatisée que le déploiement des précédentes technologies de réseaux mobiles $(1 \mathrm{G}, 2 \mathrm{G}, 3 \mathrm{G}, 4 \mathrm{G}, 4 \mathrm{G}+$, EDGE). Les perspectives liées à cette technologie semblent beaucoup plus profondes et structurantes.

Ces conditions vont donner naissance à des technologies de réseaux mobiles présente dans : le divertissement, les véhicules autonomes, la réalité virtuelle, la santé et la chirurgie à distance, et surtout l'industrie 4.0 et l'usine 4.0 que nous présentons dans les lignes suivantes.

\section{De l'analogique à l'Internet mobile}

Dans les années 1970, la $1 \mathrm{G}$ est la première génération de réseaux mobiles, elle est uniquement dédiée aux appels vocaux, elle repose sur la technologie « analogique ». Elle diffère des suivantes qui sont, elles, basées sur la technologie «numérique » pour aboutir actuellement sur la technologie 5G qui peut être considérée comme la génération de tous les usages.

Pour présenter à l'aide d'un exemple simple la différence entre la $4 \mathrm{G}$ et la $5 \mathrm{G}$, il est possible d'utiliser les propriétés de la lumière :

- La 4G peut être comparée à une lampe qui émet une lumière diffuse et qui éclaire une large surface, mais cet éclairage ne porte pas très loin, comme un parasol ;

- La $5 \mathrm{G}$ peut être comparée à une lampe dont la lumière éclaire vers un point précis mais plus loin et avec la même puissance, comme un faisceau.

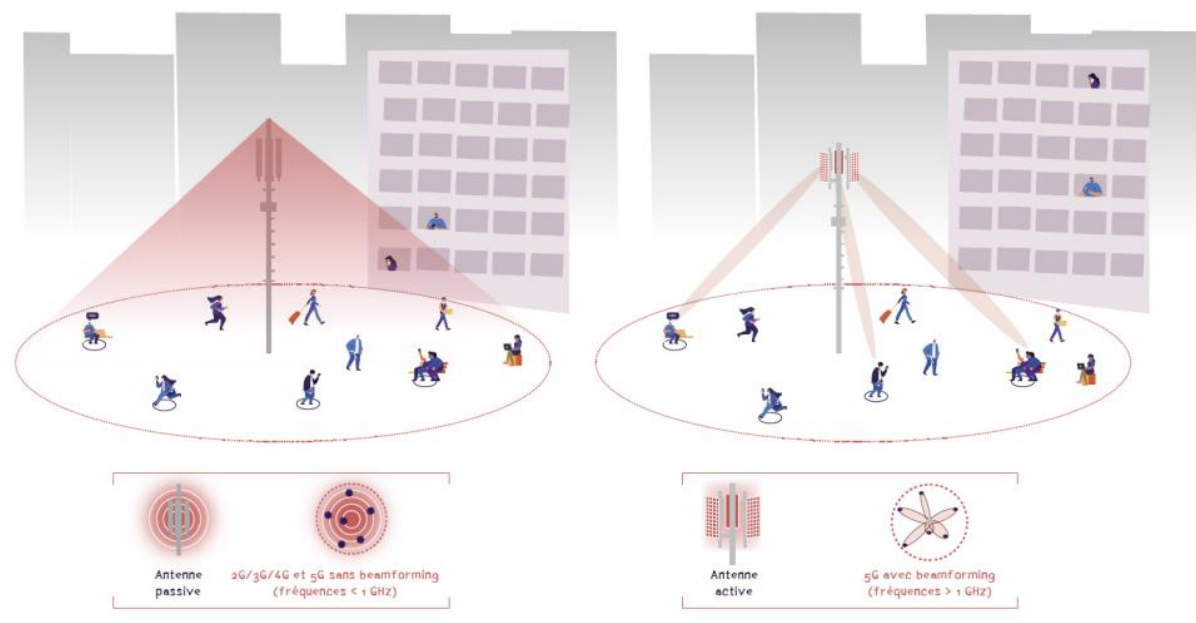

Figure 1. Les antennes actives et passives : une nouveauté de la 5G, le beamforming

Source : ACERP-2020 
L'innovation ne repose pas seulement sur la vitesse, mais également sur le débit du réseau, ainsi un grand nombre de personnes ou d'objets pourront se connecter simultanément. Le temps de réaction ou latence est un facteur très important, il est de l'ordre de la milliseconde.

\subsection{L'évolution des réseaux mobiles : traits caractéristiques}

Les Réseaux mobiles sont au cœur des débats polémiques sur la mise en place de la 5G. Mais connaissons-nous exactement les différences entre les réseaux connus sous les sigles : 1G, 2G, G, E, $3 \mathrm{G}, \mathrm{H}+$ ou $4 \mathrm{G}$ et $4 \mathrm{G}+$ ( $\mathrm{G}$ pour génération). En fait, ces sigles nous indiquent le type de réseau mobile auquel nous avons accès. Il faut savoir que cela dépend de la qualité de vos communications téléphoniques, du débit et de la performance de votre connexion Internet mobile. Au début de la mise en place du réseau mobile, le téléphone portable a utilisé tour à tour 4 générations de réseaux mobiles qui se sont succédées : 1G, 2G, 3G, 4G. Elles ont toutes leurs spécificités.

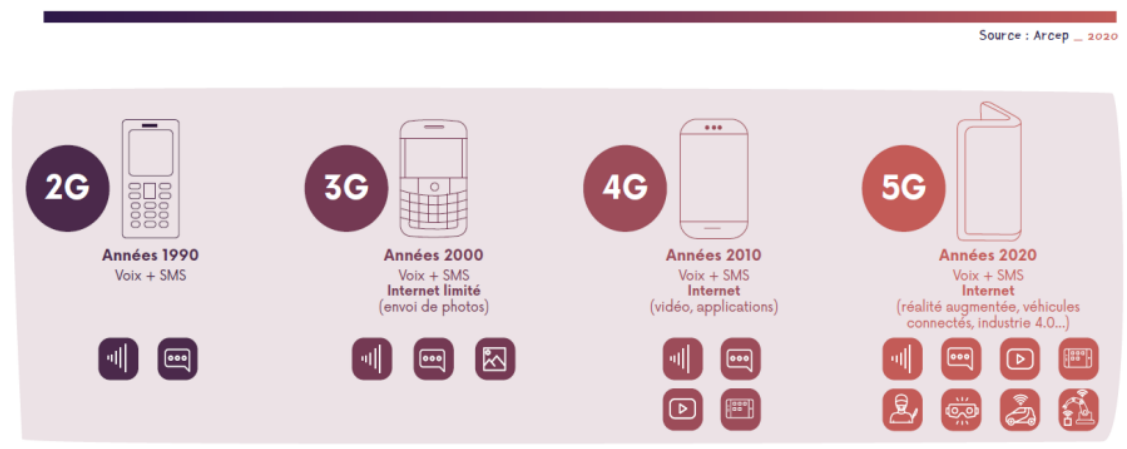

Figure 2 : Du réseau $2 G$ au réseau $4 G$

\subsubsection{La 1G, technologie analogique}

Dans les années 70, la $1 \mathrm{G}$ est la première génération de réseaux mobiles, elle est uniquement dédiée aux appels vocaux, elle repose sur la technologie « analogique ». Elle diffère des suivantes qui sontelles basées sur la technologie «numérique ». De nombreux standards du réseau $1 \mathrm{G}$ ont eu cours à travers le monde depuis son apparition en 1976. La 1G présente des défauts : une communication mobile médiocre, un service non sécurisé (appels non cryptés) et coûteux. Le réseau $1 \mathrm{G}$ a commencé à céder la place à la $2 \mathrm{G}$ dans les années 80 . Il est désormais obsolète. La France abandonne la $1 \mathrm{G}$ au profit exclusif de la $2 \mathrm{G}$ à partir de 2000.

\subsubsection{La 2G, norme européenne de téléphonie mobile.}

Le réseau 2G, apparu en 1982, est le premier réseau de téléphonie mobile en France. Celui-ci fonctionne sur des fréquences dans les bandes 900 et $1800 \mathrm{MHz}$ et c'est l'avènement des technologies de la téléphonie mobile «numérique » début avec le réseau $2 \mathrm{G}$ (deuxième génération de réseaux mobiles), déployé en France dans les années 1990, la 2G (GSM Global System for Mobile Communications) est plus fiable avec un cryptage des données transmises, le débit de transmission est de 9,6 kbps maximum. La 2G, permet de passer des appels vocaux, d'envoyer des SMS (Short Message Service), voire des MMS (Multimédia Message Service). Elle a surtout l'avantage d'être moins coûteuse pour l'utilisateur. La $2 \mathrm{G}$ est améliorée par la mise en place de deux réseaux :

- Le GPRS (General Packet Radio Service), aussi appelé 2,5G ou 2G+, améliore notablement le débit maximal de transfert de données avec un débit maximal de 171,2 Kbit/s. Il permet le transfert de données de volume modéré. C'est le précurseur sur l'internet mobile. Les téléphones portables sont aujourd'hui au moins compatibles au réseau 2,5G.

- Le EDGE (Enhanced Data Rate for GSM Evolution), c'est l'accès aux applications multimédias. Il est aussi appelé 2,75G, avec un débit maximal de $384 \mathrm{Kbit} / \mathrm{s}$. Les opérateurs comme Orange et 
Bouygues Telecom ont déployé EDGE afin de faciliter la transition entre $2 \mathrm{G}$ et $3 \mathrm{G}$. Les standards GPRS constituent de ce fait une évolution du GSM. Ils permettent un accès à l'internet et à une consultation de mails à partir d'un téléphone mobile ou d'un micro-ordinateur.

\subsubsection{La 3G démocratisation de l'Internet mobile.}

La 3G utilise la norme UMTS (Universal Mobile Telecommunications System), c'est un accès à l'Internet haut débit, entre $144 \mathrm{kbit} / \mathrm{s}$ et $2 \mathrm{Mbit} / \mathrm{s}$. Elle permet de télécharger plus rapidement des données, des applications, des jeux, d'envoyer des vidéos, de regarder des vidéos en streaming sur YouTube, ou Dailymotion, de faire de la visio-conférence, d'accéder à la TV mobile, et bénéficier du GPS. En France, le réseau 3G fonctionne sur les bandes de fréquences $900 \mathrm{MHz}$ et $2100 \mathrm{MHz}$. La 3G a connu trois évolutions avec :

- La 3G+ aussi appelée « H » pour HSPA (High Speed Packet Access), avec un débit compris entre $300 \mathrm{kbit} / \mathrm{s}$ et 14,4 Mbit/s. La 3G+ est 7 fois plus rapide que la 3G.

- Le H+ (ou HSPA+), avec un débit moyen de $5 \mathrm{Mbit} / \mathrm{s}$.

-Le H+ Dual Carrier (ou DC-HSPA+), avec un débit plafond de $42 \mathrm{Mbit} / \mathrm{s}$.

\subsubsection{La 4G le très haut débit.}

La 4G déployée en France en 2013, elle est basée sur la norme LTE (Long Term Evolution) dont le débit théorique atteint les $150 \mathrm{Mbit} / \mathrm{s}$. C'est le réseau ou l'Internet très haut débit, elle permet de transférer rapidement des fichiers volumineux (photos, musiques, vidéos, etc.), de visionner des vidéos en $\mathrm{HD}$, de faire des appels vidéo de meilleure qualité. D'après l'Autorité de Régulation des Communications Electroniques et des Postes (ARCEP), la 4G permet à « l'utilisateur de disposer d'une connexion environ 3 fois plus rapide qu'en $3 \mathrm{G} »$.

\subsection{Le débat entre la $4 G$ et $4 G+$, les premiers pas vers la $5 G$}

En 2014 la 4G+ est lancée, elle offre un débit maximal théorique de l'ordre de $1 \mathrm{Gbit} / \mathrm{s}$. Elle est basée sur la norme LTE-Advanced (Long Term Evolution Advanced), deux fois plus rapide que la 4G. La $4 \mathrm{G}+$ se connecte en simultané à plusieurs bandes de fréquences $4 \mathrm{G}$. Alors que la $4 \mathrm{G}+$ s'implante partout en France, elle va devoir faire face à l'arrivée de la 5G. La 5G ou l'ultra haut débit mobile : une innovation de rupture?

D'après l'ARCEP, la $5 \mathrm{G}$ correspond à une " génération de rupture qui permettra un saut de performance ». Elle apportera aux usagers l'ultra haut débit mobile, avec des débits dépassant les 10 Gbit/s ! Les opérateurs, Orange, SFR ou encore Bouygues Telecom rivalisent déjà dans leurs effets d'annonce.

L'objectif de la Commission européenne est la couverture d'au moins une grande ville de chaque Etat membre dès 2020. Dans son panorama des expérimentations réalisées par l'Arcep la 5G est dans sa phase expérimentale dans certaines villes françaises : Belfort, Bordeaux, Douai, Grenoble, Lannion, Lille, Lyon, Marseille, Nantes, Toulouse, Sophia-Antipolis et en Île-de-France.

\section{Le déploiement de la 5G}

Tristan Gaudiaut, en avril 2019, écrit «La révolution technologique de la 5G, les premières régions du monde seront couvertes par la 5G. Cette nouvelle génération de téléphonie mobile occupera une place très importante dans l'avenir ». 


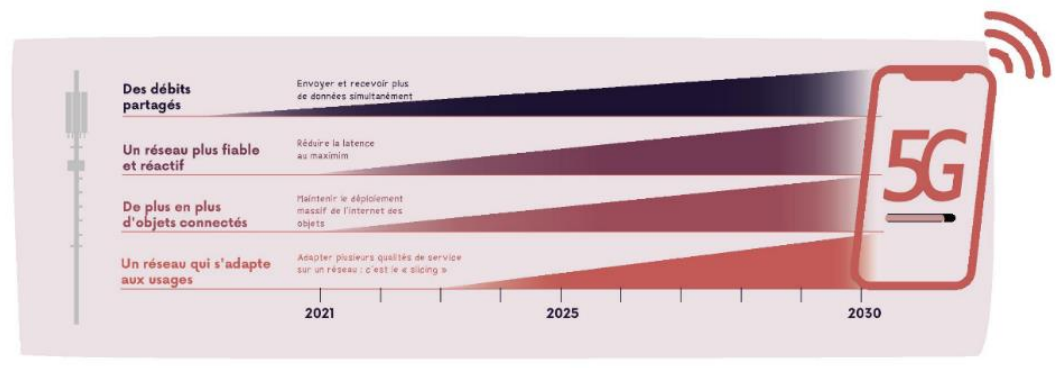

Figure 3. Que permettra la 5g. Une technologie évolutive

La 5G promettra de décupler la vitesse de téléchargement effective, tout en divisant par dix le temps de latence. La bande passante de la $5 \mathrm{G}$ sera également beaucoup plus élevée, tout comme le nombre de porteuses qui passeront à 16 contre 5 avec les normes 4G/LTE (4G+). Ces améliorations sont cruciales pour le développement des services connectés comme : villes intelligentes, big data, maison connectée, conduite autonome, ...

Les États-Unis et la Corée du Sud sont engagés dans la course pour être les premiers à offrir la $5 \mathrm{G}$ à leurs citoyens. Déjà opérationnel pour les entreprises depuis décembre, les opérateurs de téléphonie mobile sud-coréens prévoient l'accessibilité du réseau $5 \mathrm{G}$ au grand public d'ici la mi-avril. Tandis que le fournisseur américain Verizon a annoncé vouloir lancer son offre 5G le 11 avril.

D'après l'étude réalisée par Statista ${ }^{1}$, les premiers forfaits $5 \mathrm{G}$ commencent progressivement à être annoncés en France. Orange a donné le coup d'envoi début octobre, en dévoilant des tarifs allant de $24,99 € /$ mois (70 Go de données) à 79,99 €/mois (données illimitées) pour la première année. Ceci dit, la nouvelle génération de réseau mobile reste encore très embryonnaire et les forfaits ne devraient pas être d'une très grande utilité pour la majorité des usagers lors du lancement commercial

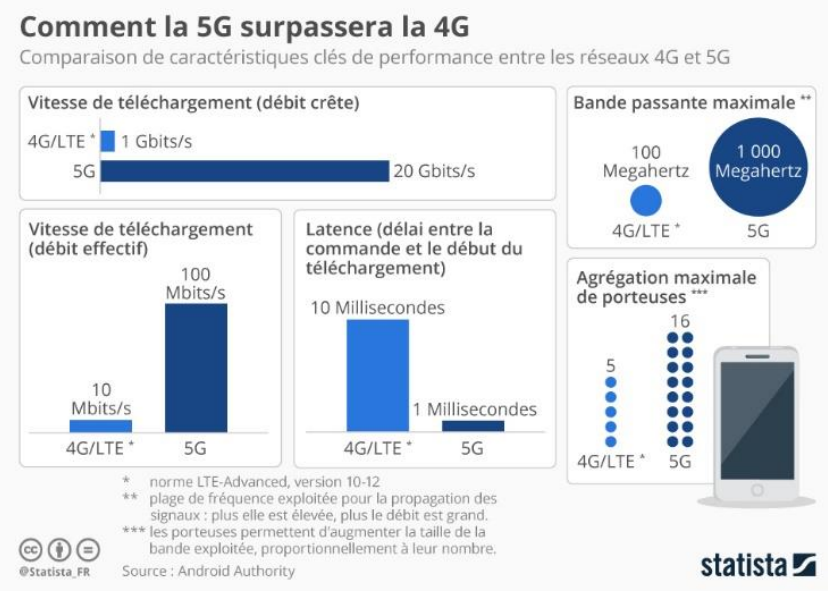

Figure 4. Comment la $5 G$ surpassera la $4 G$

\footnotetext{
${ }^{1}$ Statista est un portail en ligne allemand offrant des statistiques issues de données d'instituts, d'études de marché et d'opinion ainsi que de données provenant du secteur économique.
} 
Une étude réalisée par Open Signal ${ }^{2}$ dans les pays où la technologie est déjà disponible donne un aperçu de son potentiel. En moyenne, sur la totalité des pays étudiés, la vitesse de téléchargement de la $5 \mathrm{G}$ était cinq à six fois plus rapide que la $4 \mathrm{G}$. Mais comme le montre notre infographie, d'énormes différences existent entre les pays, en fonction notamment de l'état de déploiement de cette technologie.

Ce sont les utilisateurs d'Arabie saoudite qui bénéficient actuellement des vitesses moyennes de téléchargement 5G les plus rapides : environ 377 mégabits par seconde, soit un peu plus que les 336 mégabits par seconde mesurés en Corée du Sud. La vitesse moyenne relativement modeste relevée aux États-Unis (52 mégabits/seconde) est en grande partie due au niveau de développement du réseau et aux spectres de fréquences actuellement utilisés par les opérateurs. Comme le souligne les analystes d'Open Signal, la vitesse de téléchargement n'est pas le seul élément de performance à prendre en compte. Le temps pendant lequel les utilisateurs ont la possibilité d'être connectés à la $5 \mathrm{G}$ est également extrêmement important.

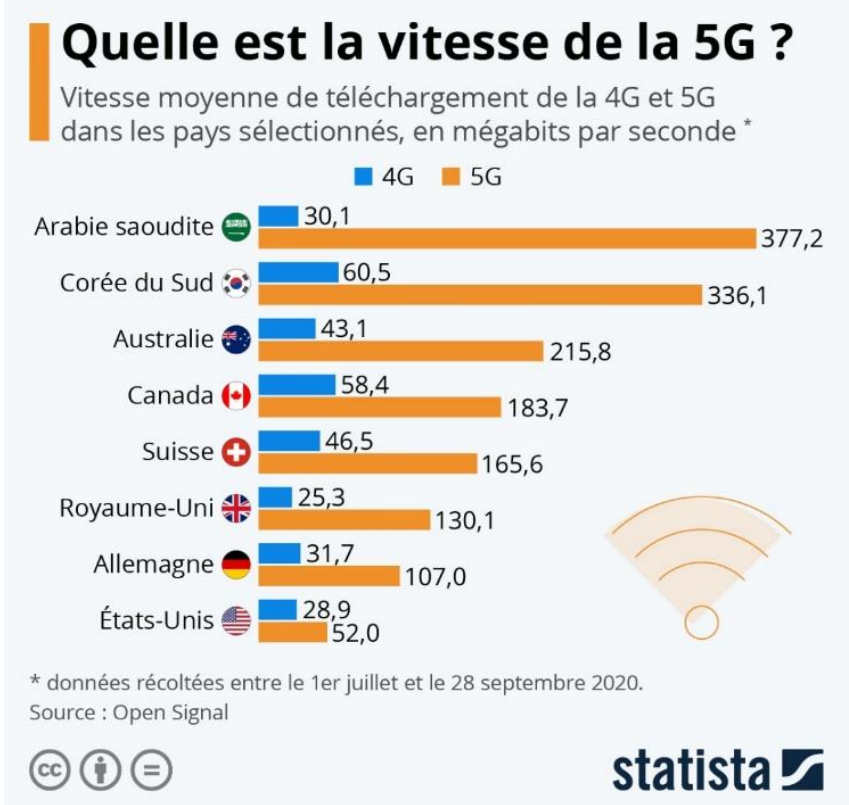

Figure 5. Quelle vitesse de la 5G

En mai 2018, Pascaline Boittiaux, analyste chez Statista, étudie «Le marché du smartphone en attente de la 5G ». Les ventes de smartphones accusent un recul en 2018, selon les prévisions du cabinet IDC ${ }^{3}$. Seuls 1,46 milliard de smartphones s'écouleront en effet cette année, soit une baisse de $0,2 \%$ par rapport à 2017, largement due à un repli du marché chinois.

Mais la tendance devrait de nouveau être à la hausse l'année suivante et le cabinet IDC prévoit même une croissance annuelle d'environ $3 \%$ à partir de 2019 , si bien que les ventes dépasseront les 1,65 milliard de smartphones en 2022. Ce rebond de la croissance sera soutenu par l'arrivée de la 5G, qui nécessitera l'achat de smartphones compatibles avec l'Internet ultra-rapide.

\footnotetext{
${ }^{2}$ Opensignal est une société d'analyse mobile indépendante spécialisée dans la " quantification de l'expérience de réseau mobile ». Cette société combine des mesures du monde réel avec une analyse scientifique pour fournir des informations indépendantes sur la connectivité mobile à l'échelle mondiale. Le site https://www.opensignal.com

${ }^{3}$ IDC est un acteur majeur de la Recherche, du Conseil et de l'Évènementiel sur les marchés des Technologies de l'Information, et des Télécommunications.
} 


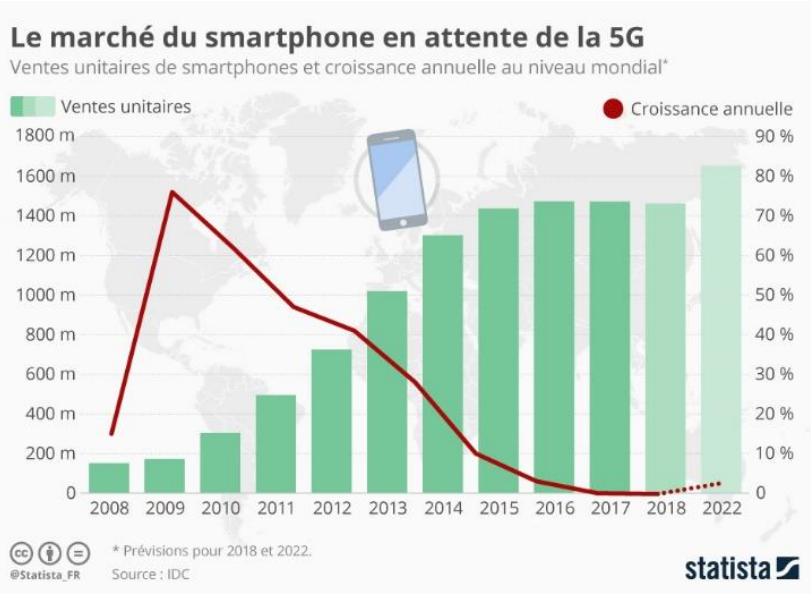

Figure 6. Le marché du smartphone

\section{La diffusion de la $5 \mathrm{G}$ et son potentiel de changement}

\subsection{Le divertissement}

Aujourd'hui 50\% des vidéos sont regardées sur un smartphone, le streaming haute définition va s'accentuer. La retransmission d'événements en direct, la télévision en HD seront accessible sans interruption depuis un appareil mobile. L'industrie du divertissement va changer. Pour les jeux vidéo, la réalité virtuelle ou augmentée permettra de créer des expériences immersives.

\subsection{La voiture autonome}

Les véhicules seront les prochains objets connectés. Cette technologie «vehicule to vehicule » (V2V) sera l'innovation la plus importante en matière de sécurité sur la route.

Ericsson et le fournisseur de services nordique Telia ont donné vie à des véhicules guidés automatisés, à la réalité augmentée (RA) et à un grand nombre de capteurs dans l'usine de fabrication d'Ericsson à Tallinn, en Estonie, via un réseau cellulaire dédié.

Les essais de véhicules entièrement autonomes tels que la Waymo de Google, la Vision iNext de BMW et les modèles Tesla sont novateurs. Mais la voiture connectée doit réagir en temps réel aux événements qui l'entourent et cette capacité dépend des niveaux de latence et donc les évolutions de ces voitures entièrement autonomes dépendront de la 5G. En outre, le futur véhicule à conduite autonome génèrera de grandes quantités de données pour la détection et la communication avec son environnement.

\subsection{La réalité augmentée et réalité virtuelle et la 5G}

D’après SFR Business, Réalité Virtuelle, Réalité Augmentée et Réalité Mixte vont connaître un bouleversement avec le déploiement de la 5G. Si la réalité virtuelle est capable de nous transporter dans un autre monde, la réalité augmentée est destinée à enrichir l'existant en superposant des informations complémentaires.

- Le principe de la réalité augmentée consiste à « superposer des informations virtuelles sur le monde réel via un terminal connecté (smartphone ou tablette, lunettes, casque) »;

- Le principe de la réalité virtuelle, consiste en une technologie immersive qui utilise un casque connecté qui projette une simulation modélisée en 3D ;

- Le principe de la réalité mixte consiste à utiliser les avantages des deux technologies, réalité augmentée et réalité virtuelle, permettant de fusionner les deux réalités pour simuler de véritables univers alternatifs inspirés du réel. 
Ces technologies qui fonctionnent grâce à Internet et au réseau mobile $4 \mathrm{G} / 4 \mathrm{G}+$ vont voir leurs usages démultipliés grâce à un surcroît de vitesse et à une latence réduite offerts par la $5 \mathrm{G}$, dans de nombreux secteurs d'activité tel que l'aéronautique, l'industrie, l'automobile, la santé

\subsection{La santé}

D’après Anne Perrin, spécialiste du risque électromagnétique, « Les niveaux ambiants sont bien inférieurs aux valeurs limites réglementaires, elles-mêmes bien au-dessous des seuils d'apparition d'effets sur la santé ». Le réseau s'appuiera sur une bande de fréquences très haute : $26 \mathrm{GHz}$. Mais plus la fréquence est haute, plus la portée des ondes est courte. Cela nécessite de multiplier les antennes ! En 2018, sur les quelques 3000 mesures réalisées en France par l'ANFR, 99\% étaient inférieures à 6,1 $\mathrm{V} / \mathrm{m}$ (les limites réglementaires varient entre $28 \mathrm{~V} / \mathrm{m}$ et $87 \mathrm{~V} / \mathrm{m}$ selon les fréquences). Un organisme indépendant, reconnu par l'Organisation mondiale de la Santé (OMS), contrôle les limites d'expositions, qui ensuite sont validées par un comité scientifique européen et par l'Agence de sécurité sanitaire de chaque pays, en France par l'Agence nationale des fréquences (ANFR). "Les risques sanitaires varient selon le degré d'absorption des fréquences par le corps. Plus la fréquence des ondes électromagnétiques augmente, plus les ondes sont absorbées par les couches superficielles de la peau. Ce qui fait diminuer la profondeur de pénétration de ces ondes dans l'organisme ».

\subsubsection{La santé et la chirurgie à distance}

Les appareils portables avec leurs applications, vont permettre des consultations en ligne sécurisées. La chirurgie robotique à distance va pouvoir se développer et permettre la prise en charge des patients en dehors de l'environnement hospitalier, la 5G réalisera des économies sur le temps de consultation chez le médecin ou à l'hôpital. C'est également des économies en nombre de trajets pour consulter. On peut également citer :

- Une surveillance en temps réel des patients grâce aux objets connectés ;

- Le contact des patients à tout moment, selon leur besoin de santé, avec leur médecin ;

- La $5 \mathrm{G}$ réalisation des opérations chirurgicales à distance, pour des millions de personnes dans le monde entier.

Mais les applications les plus prometteuses seront dans l'industrie pour accélérer sa digitalisation et gagner en compétitivité. Selon les derniers chiffres publiés par France Industrie, 25 milliards d'euros sont investis chaque année par le secteur de l'industrie en R\&D.

\subsection{L'environnement}

Certains s'inquiètent sur la consommation énergétique induite par la 5G. L'accès plus rapide aux données compenserait pour certains des économies d'énergie réalisées ! Quant à l'impact environnemental de la $5 \mathrm{G}$ celui-ci est plus compliqué à mesurer. On peut mettre en avant la baisse des émissions de CO2 entraînée par l'essor du télétravail ! Le Sénat a rendu, mercredi 24 juin, 25 propositions pour réduire l'impact environnemental du numérique. Parmi les mesures-phare, l'interdiction des forfaits mobiles illimités.

Des millions d'appareils 4G vont être mis au rebus prématurément, au bénéfice d'appareils flambants neufs et compatibles 5G. smartphones, tablettes, .... Selon un rapport d'Ericsson, on comptait déjà 13 millions d'abonnés à la $5 \mathrm{G}$ d'ici fin décembre et le milliard devrait être atteint d'ici 2023. Le cabinet d'études IDC prévoit que le marché smartphone va retrouver une embellie. Les appareils équipés pour la $5 \mathrm{G}$ devraient représenter $9 \%$ du total des ventes dès 2020 et passer à $30 \%$ en 2023, de quoi franchir à nouveau la barre record des 1,4 milliards de smartphones écoulés en une année. A cela, devrait s'ajouter l'augmentation du nombre d'objets connectés de type montres « intelligentes ». 


\subsection{L'industrie}

Les objets connectés «Internet of Things » (IoT) concernent également l'industrie ; ces capteurs intelligents permettent un meilleur contrôle de la sécurité des bâtiments et permettent la mise en place d'une maintenance prédictive. La logistique, la gestion des stocks, le suivi en temps réel seront bénéficiaires de la 5G. L’Usine du futur sera rapide et flexible.

Steven Heckler, expert des questions technologiques au $\mathrm{BDI}^{4}$ (la fédération de l'industrie allemande), une nouvelle phase industrielle arrive « La question des infrastructures $5 \mathrm{G}$ est un sujet extrêmement délicat », il fait remarquer également qu'il faut « Travailler à renforcer la souveraineté technologique européenne, mais cela ne signifie pas mettre fin à la mondialisation ». Il faut des exigences de sécurité, que tous les fabricants doivent respecter. Il continue en expliquant « qu'il est nécessaire de rester en mesure d'évaluer des technologies venues de pays tiers comme la Chine, au même titre que les offres des fournisseurs européens », comme Nokia et Ericsson. C'est la raison pour laquelle la notion de «souveraineté » défendue par Emmanuel Macron est volontiers reprise dans les milieux économiques allemands.

\subsubsection{L'industrie 4.0 ou l'usine 4.0}

Les 28 et 29 septembre 2017, l'Estonie a accueilli, dans sa capitale Tallinn, le premier sommet européen dédié aux questions numériques, les chefs d'État et de gouvernement ont notamment examiné les propositions récentes de la Commission européenne en matière de cybersécurité et de taxation de l'économie numérique. Lors de ce sommet, Bosch et Nokia ont fait une démonstration conjointe de l'utilisation de la $5 \mathrm{G}$ dans la production, et en particulier dans l'environnement émergent des usines connectées, ou usine 4.0. La vitesse, les performances en temps réel et la fiabilité de transmission de la $5 \mathrm{G}$ vont transformer la communication mobile en général, mais sans doute plus encore le monde industriel, la quatrième révolution industrielle.

\subsubsection{Réalité augmentée pour le dépannage}

L'usine Ericsson de Tallinn est la première usine à utiliser la réalité augmentée pour le dépannage afin de réduire le coût des pannes et réduire les temps d'arrêt de production. L'usine utilise également la RA pour détecter les inefficacités opérationnelles telles que la planification de la maintenance imparfaite, les diagnostics de panne, mais aussi pour la formation. À ce jour, Ericsson de Tallinn, réalise des gains de temps allant jusqu'à $50 \%$.

\subsubsection{Exemples: Bosch et l'usine 4.0}

Le Dr Andreas Müller, chercheur chez Bosch et président de l'initiative internationale 5G-ACIA, affirme que « Nous pensons que la $5 \mathrm{G}$ va bouleverser les process de fabrication », et « qu'elle pourrait même modifier la manière dont les usines sont construites. ". L'industrie 4.0 apporte davantage d'intelligence et de flexibilité à la production. L'Usine du futur sera avec une vitesse plus élevée, une capacité en temps réel accrue et une flexibilité améliorée, plusieurs applications industrielles deviennent beaucoup plus faciles à envisager :

-L'interaction entre les machines est une notion essentielle pour accroître la distribution de l'intelligence dans les usines ;

- Les boucles de régulation nécessitent un contrôle en temps réel ;

- Les capteurs sans fil permettent de mieux contrôler la production en temps réel ;

- Les véhicules autonomes utilisent l'intelligence basée dans le cloud pour fournir les matériaux en juste-à-temps dans l'usine ;

\footnotetext{
${ }^{4}$ https://business.twitter.com/fr/success-stories/bdi-success-story.html
} 
- La réalité augmentée permettra de visualiser en temps réel et directement sur la machine son état de fonctionnement ;

- Le découpage des réseaux, permet aux industriels de définir plusieurs réseaux ;

- Les industriels pourront mettre en place « l'Edge Computing » ${ }^{5}$.

\subsubsection{Exemple : « Factory $56 »$}

Jörg Burzer Membre du Conseil divisionnaire de gestion des voitures Mercedes-Benz, en collaboration avec la société de télécommunications Telefónica Deutschland et le fournisseur de réseau Ericsson, met en place le premier réseau mobile $5 \mathrm{G}$ au monde pour la production automobile dans son « Factory 56 » à Sindelfingen. Avec la « Factory 56 », « nous ne construisons pas seulement la production de voitures intelligentes du futur, mais nous mettons également en place le premier réseau mobile 5G au monde dans une usine d'assemblage de véhicules ».

\subsubsection{Exemples d'applications $5 \mathrm{G}$ en industrie :}

Avec une vitesse plus élevée, une capacité en temps réel accrue et une flexibilité améliorée, plusieurs applications industrielles deviennent beaucoup plus faciles à envisager :

-L'interaction entre les machines est une notion essentielle pour accroître la distribution de l'intelligence dans les usines ;

- Les boucles de régulation qui nécessitent un contrôle en temps réel très fiable et un cycle très court peuvent fonctionner dans leur tranche de réseau sans subir l'effet perturbateur ;

- Les capteurs sans fil permettent de mieux contrôler la température de service, les vibrations et les émissions sonores sur les machines de production, déclenchant une alerte si l'un des paramètres dépasse les seuils fixés dans un laps de temps donné.

\section{La 5G : une affaire mondiale !}

La Corée du Sud a été la première à déployer un réseau $5 \mathrm{G}$ et elle devrait rester en tête en ce qui concerne la pénétration de cette technologie. D'ici 2025, il est attendu que près de $60 \%$ des abonnements de téléphonie mobile en Corée du Sud soient des forfaits 5G. Concernant la vitesse de connexion, le pays est actuellement devancé par l'Arabie Saoudite, selon les données d'Open Signal.

Le GSMA ${ }^{6}$ estime que la $5 \mathrm{G}$ couvrira $34 \%$ des connexions mobiles à l'horizon 2025. Plusieurs pays européens disposent déjà d'un réseau 5G opérationnel comme la Finlande, la Suisse, le RoyaumeUni, l'Allemagne, l'Espagne ou encore l'Italie. En France, les enchères pour l'attribution des fréquences $5 \mathrm{G}$ se dérouleront en octobre ou novembre avec une commercialisation qui devrait débuter à la fin de l'année 2020.

\footnotetext{
${ }^{5}$ Définition : modèle d'informatique distribuée dans lequel l'informatique se déroule près de l'endroit physique où les données sont collectées et analysées pour procéder au traitement des données en périphérie du réseau, à proximité de la source, au cœur même des usines. Le site https://www.startus.cc/

${ }^{6}$ La GSM Association (Global System for Mobile Communications) représente les intérêts des opérateurs de téléphonie mobile du monde entier, réunissant plus de 750 opérateurs et près de 400 entreprises dans l'écosystème mobile au sens large. Le site
} 


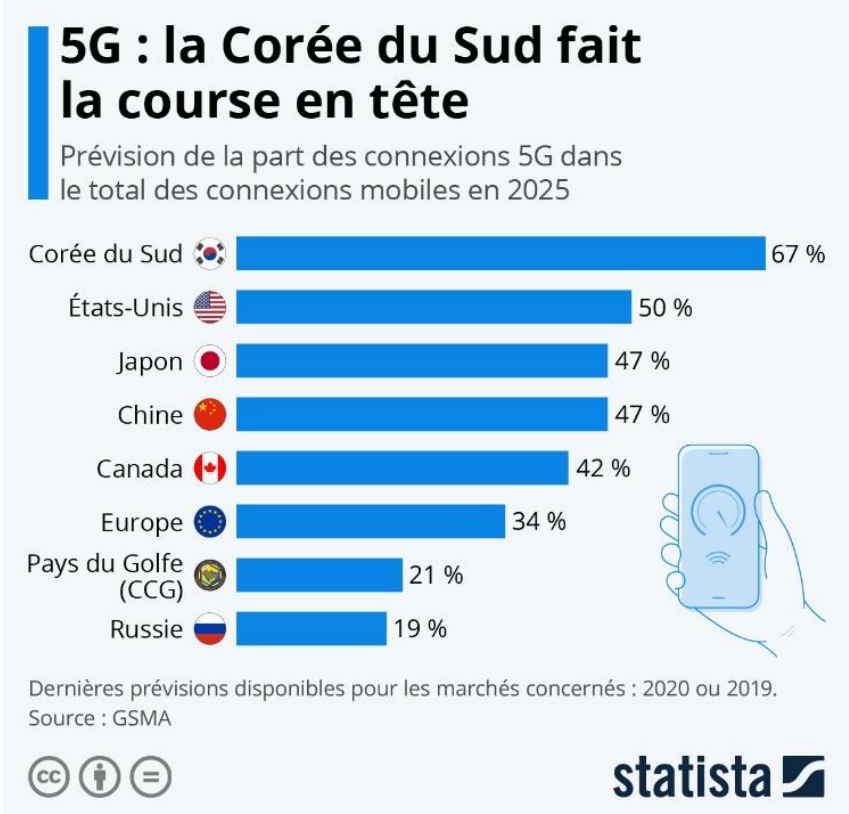

Figure 7. La Corée du sud en tête

Aux États-Unis, la répartition de l'utilisation des bandes de fréquences radio n'est pas la même qu'en Europe. La bande 3,5 $\mathrm{GHz}$ est notamment utilisée par l'armée, ce qui interdit a priori dans l'immédiat d'en faire usage pour le déploiement de la 5G. Ainsi, les États-Unis ont choisi pour la 5G d'utiliser la bande 24 - $28 \mathrm{GHz}$, qui permet de meilleurs débits, mais avec des portées plus faibles.

Rien n'arrête la 5G en Chine, pas même le coronavirus, ni les nouvelles sanctions américaines contre Huawei. Pour la première fois, la deuxième économie mondiale vient même de franchir la barre symbolique des 100 millions d'abonnés 5G, selon les derniers chiffres publiés par China Mobile et China Telecom, deux des trois opérateurs télécoms chinois. Dans le détail, China Mobile a raflé la mise avec 70 millions d'abonnés $5 \mathrm{G}$, soit dix fois plus qu'il y a six mois.

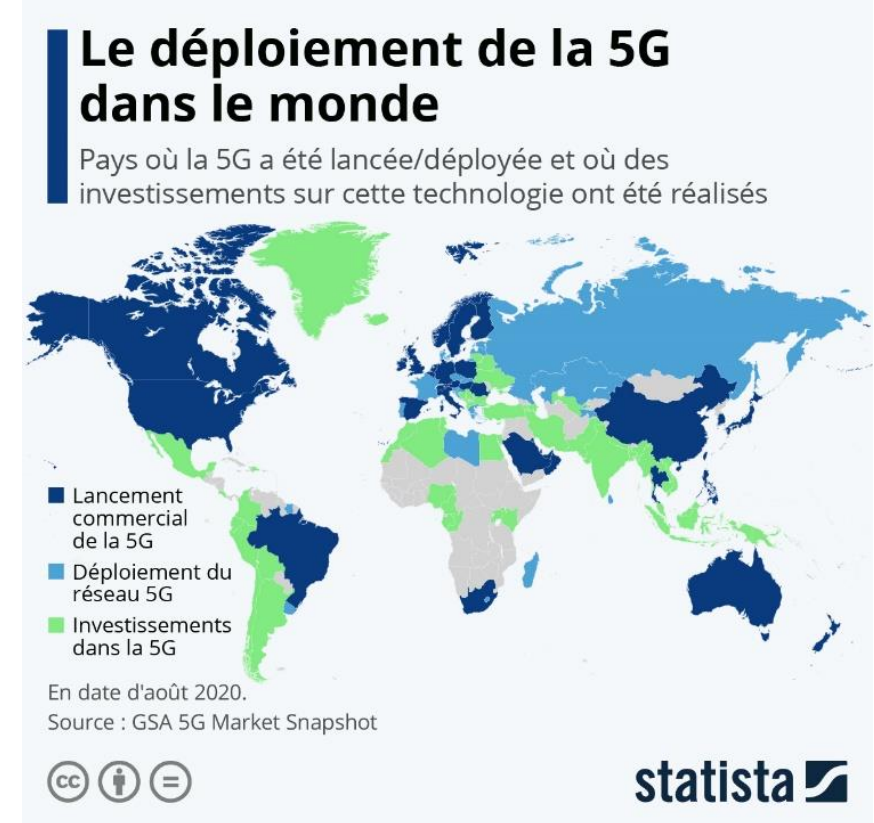

Figure 8. Le déploiement de la $5 G$ dans le monde

Le déploiement de la $5 \mathrm{G}$ fait l'objet de tensions internationales, entre les États-Unis et la Chine, sur fond de questions de souveraineté nationale et de guerre commerciale 


\section{La G5 en Europe}

En Octobre 2019 L'ANSES édite un rapport sur « Exposition de la population aux champs électromagnétiques liée au déploiement de la technologie de communication « $5 \mathrm{G}$ » et effets sanitaires associés ». Entre autres, elle fait le point sur la mise en place en Europe et dans le monde de l'implantation de la 5G. Un petit aperçu :

- Au Royaume-Uni, l'opérateur britannique EE a confirmé le lancement de services commerciaux Rapport préliminaire Comité d'experts spécialisé : «Agents physiques, nouvelles technologies et grands aménagements » Octobre 2019 5G le 30 mai 2019

- En Suisse, certains opérateurs ont également confirmé le lancement de la 5G. Un communiqué d'avril 2019 évoque la couverture de 54 localités par Swisscom, qui compterait augmenter la couverture jusqu'à $90 \%$ de la population d'ici fin 2019.

- En Italie, Telecom Italia (TIM) a lancé des services commerciaux 5G après l'activation de son réseau dans certaines parties de Rome et de Turin le 24 juin 2019.

- La Finlande, l'Irlande, 1'Espagne ou encore 1'Estonie disposeraient également de réseaux 5G dans certaines villes

- La Pologne a attribué les bandes de fréquences nécessaires à la 5G et Ericsson et Orange ont lancé des tests $5 \mathrm{G}$ dans la région de Varsovie

- En Allemagne, l'agence fédérale allemande des réseaux a annoncé que la vente aux enchères des bandes de fréquences de la 5G, commencée en mars 2019, avait abouti au total à 6,55 milliards d'euros répartis entre quatre opérateurs : Deutsche Telekom, Vodafone Allemagne, Telefonica et 1\&1 Drillisch.

Tristan Gaudiaut, analyste chez statista, propose une carte et une analyse sur le déploiement de la 5G en Europe. Les quatre opérateurs lauréats : Orange, SFR, Bouygues Telecom et Free Mobile ont engagé 2,8 milliards d'euros. Le lancement commercial de la 5G est prévu en France vers la fin de l'année.

Ci-dessous la carte proposée par Ookla ${ }^{7}$. Elle permet de voir où la $5 \mathrm{G}$ était déjà disponible dans 19 pays en Europe au début du mois d'octobre. Le déploiement de la 5G est relativement faible par rapport à tous ses voisins européens. Mais il faut souligner que près de 500 antennes sont actuellement testées en attendant le lancement de l'offre commerciale. Une carte présentant un état des lieux des lancements commerciaux de la $5 \mathrm{G}$ en Europe au mois d'octobre est également disponible ici.

\footnotetext{
${ }^{7}$ https://www.speedtest.net/fr
} 


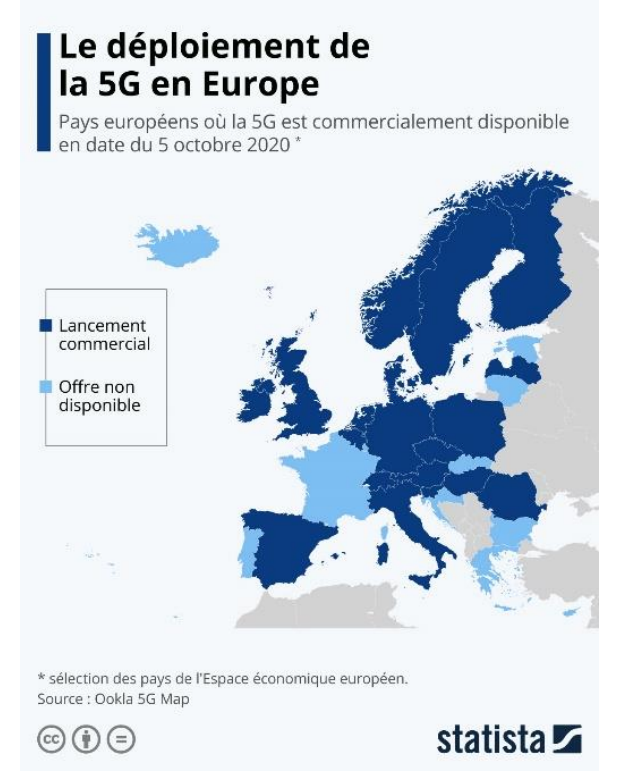

Figure 9. Déploiement de la 5Gen Europe

\section{La $5 G$ en France}

C'est une question de compétitivité, la $5 \mathrm{G}$ a de grandes applications industrielles : comme la voiture connectée, les opérations chirurgicales à distance, ...Il est évident que les entreprises françaises souhaitent suivre le rythme de la concurrence internationale, ainsi pour eux le développement de la 5G est primordial. Le secrétaire d'Etat au Numérique, Cédric O, juge la $5 \mathrm{G}$ « indispensable à la compétitivité économique de la France », tandis que le ministre de l'Economie, Bruno Le Maire ${ }^{8}$, estime que « ce serait une erreur dramatique de s'en priver ». D'autant plus que la France n'est pas en avance. Le rapport remis au gouvernement met en évidence le « relatif retard » de la France aussi bien pour l'attribution des fréquences que pour le lancement commercial du réseau $5 \mathrm{G}$ des opérateurs

En France la 5G est déployée fin novembre 2020. Parmi les villes françaises nous avons noté : Marseille avec un total de 95 antennes, Paris et Montpellier 58 et 51 antennes. Orange a réalisé un grand nombre de tests d'antennes 5G durant le mois de novembre 2020.

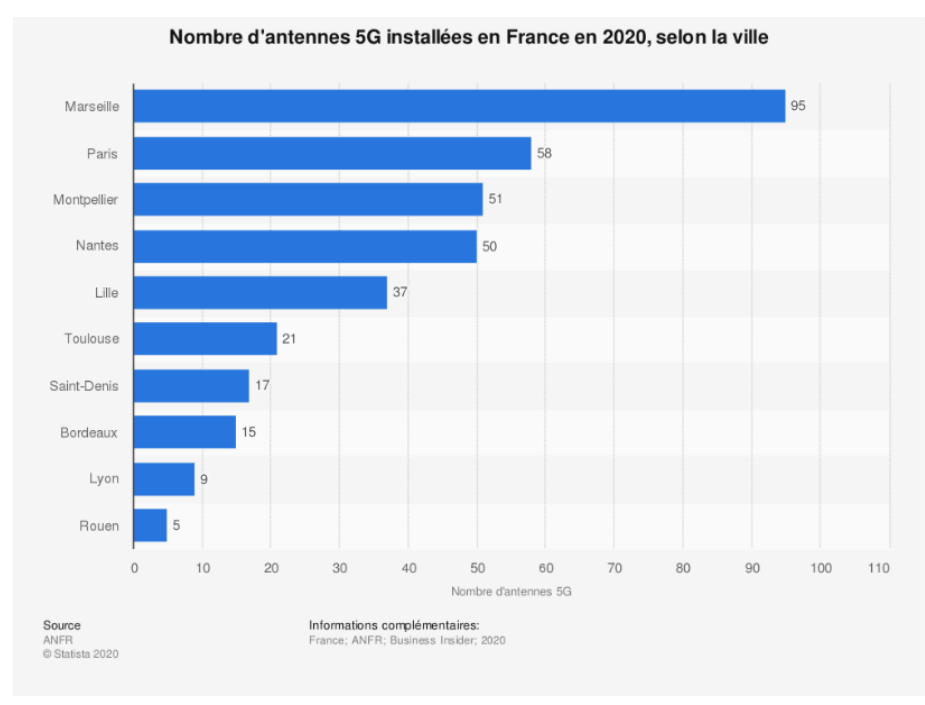

Figure 10. Nombre d'antennes 5G par ville en France 2020

\footnotetext{
${ }^{8}$ Le débat sur la $5 \mathrm{G}$ en cinq questions : sur le site https://www.lesechos.fr/tech-medias/hightech/le-debat-sur-la-5g-en-cinqquestions- 1242940
} 


\section{La 5G : relevons les défis9}

Selon une enquête menée par le Business Performance Innovation Network (BPI), les secteurs les plus impactés par la $5 \mathrm{G}$ auront une importance majeure dans de nombreux domaines comme par exemple :

- L'industrie automobile où cette nouvelle technologie devrait avoir des effets de rupture ;

- Les services de cloud computing;

- La production.

Les experts prévoient que pour un certains de domaines, seront en revanche les moins touchés

- Les services financiers,

- Le gouvernement et

- L’agriculture

Les vitesses de téléchargement, la faible latence vont jouer un rôle important pour des applications où le temps est un facteur critique, comme la conduite autonome ou la santé connectée.

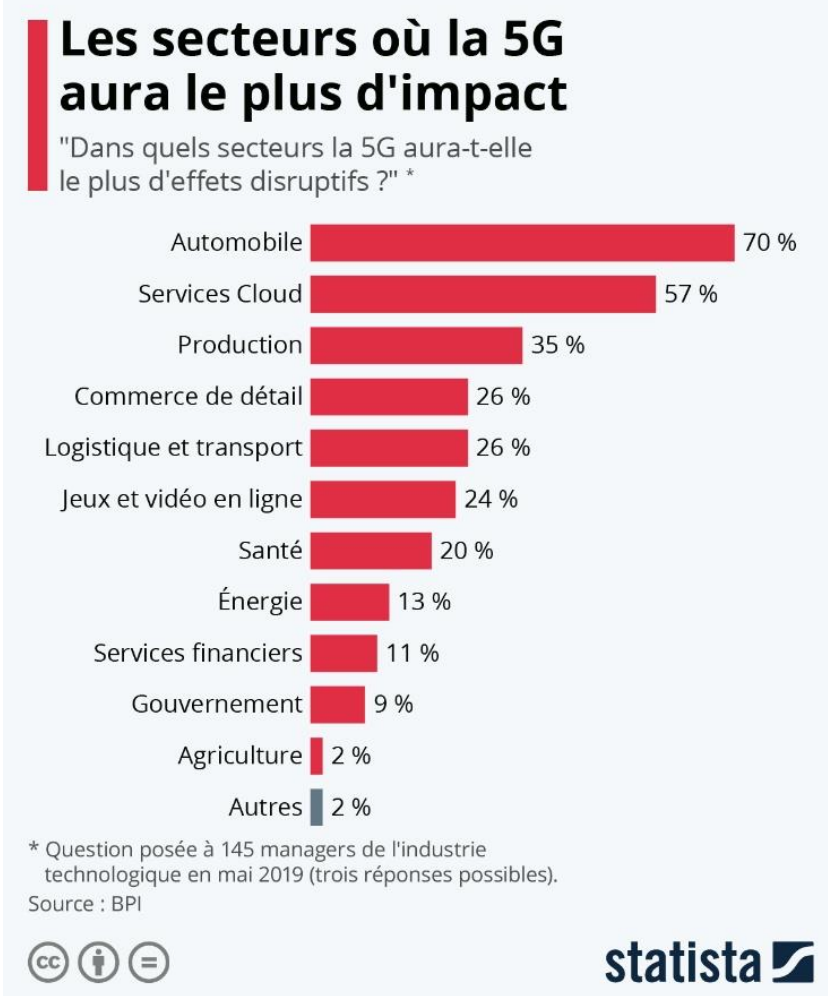

Figure 11. Les secteurs impactés par la 5G

\subsection{Le déploiement de l’internet mobile}

À l'échelle mondiale, la 5G se développe à vive-allure, selon un rapport récemment publié par $\mathrm{GSA}^{10}$, la nouvelle technologie de communication mobile était commercialement disponible dans une quarantaine de pays fin août et le réseau était en cours de déploiement dans de nombreux autres. Les

\footnotetext{
${ }^{9}$ https://www.anses.fr/fr/content/la-technologie-5g

${ }^{10}$ L'Agence GNSS européenne (GSA) publie le 3e rapport sur la technologie des utilisateurs GNSS. Le site :

https://www.gsa.europa.eu/
} 
dernières prévisions indiquent que la $5 \mathrm{G}$ pourrait atteindre le milliard d'utilisateurs dans trois ans et demi, contre quatre ans pour la $4 \mathrm{G}$ et douze ans pour la $3 \mathrm{G}$.

L'Amérique du Nord, l'Asie de l'Est et l'Europe sont à l'avant-garde du déploiement de la 5G. Les investissements pour déployer cette nouvelle génération de réseau mobile sont réalisés dans presque tous les pays d'Asie du Sud/Sud-Est ainsi qu'en Amérique du Sud.

Selon le dernier Ericsson Mobility Report, le marché mondial de la 5G devrait compter 213 millions d'abonnements à la fin de l'année, dont plus de 190 millions rien qu'en Asie. Le nombre d'utilisateurs est amené à décoller l'année prochaine avec le déploiement de cette technologie dans d'autres régions et le cap du milliard d'abonnements dans le monde devrait être dépassé à l'horizon 2022. La plupart d'entre eux, autour de 900 millions, seront basés dans la région Asie-Pacifique, alors qu'environ 122 millions et 87 millions d'abonnements sont respectivement prévus à cette date en Amérique du Nord et en Europe.

Selon les prévisions, la $5 \mathrm{G}$ pourrait couvrir jusqu'à $65 \%$ de la population mondiale à la fin de l'année 2025 et traiter $45 \%$ du trafic mondial de données mobiles. En Corée du Sud, où la technologie est déjà en service depuis avril 2019, près de huit millions d'abonnements étaient enregistrés fin juillet auprès des opérateurs du pays.

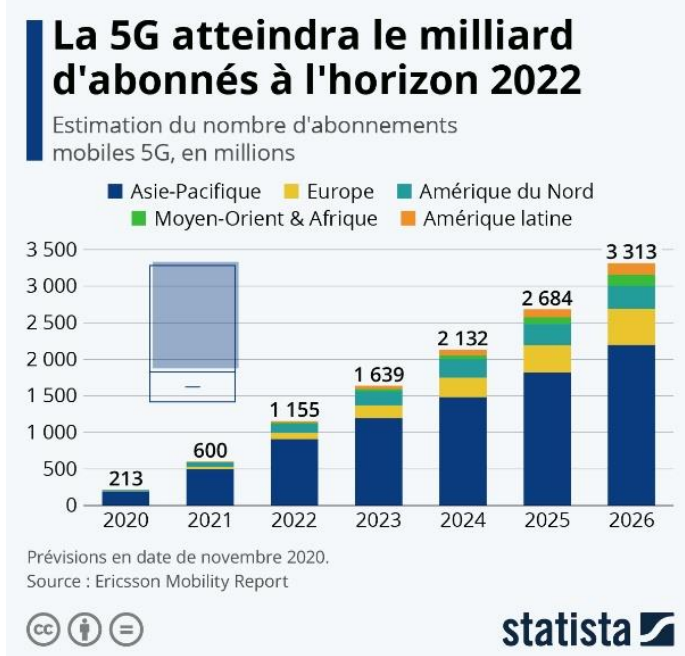

Figure 12. Le nombre d'abonnés en 2022 (prévision)

\subsection{La 5ième génération : que faut-il en penser " innovation de rupture "?}

Les réseaux mobiles déployés jusqu'à maintenant ont permis d'être connecter, de se rapprocher les uns des autres de plus en plus rapidement par : téléphonie vocale, transmission de la voix, haut débit, de faciliter la transmission de données, ... pour la génération 5G les ambitions sont encore plus grandes. Nous sommes en face d'une transformation structurelle et donc d'une innovation de rupture pour notre société.

Frédéric Bordage, expert numérique, pose question de savoir s'il était vraiment utile de déployer la $5 \mathrm{G}$ pour tous. "Est-ce qu'on prend tous ces risques pour regarder des vidéos en streaming $4 \mathrm{~K}$ à $300 / \mathrm{km}$ dans un TGV ? C'est ça le progrès de la $5 \mathrm{G}$ ? $"{ }^{11}$. Faudrait-il réserver la $5 \mathrm{G}$ aux entreprises puisque ce sont elles qui vont en tirer le plus d'applications ? Grâce au « network slicing », qui correspond à un découpage du réseau en tranches logicielles, il serait alors possible de donner priorité à

\footnotetext{
${ }^{11}$ https://www.01net.com/actualites/pourquoi-la-5g-est-une-mauvaise-nouvelle-pour-l-environnement-1846716.html
} 
certains usages critiques et permettre le développement des innovations comme : les opérations chirurgicales, la voiture autonome, ...

\subsection{L'avenir et la technologie mobile}

Selon Pierre Benhamou ${ }^{12}:$ «En France, dirigeants politiques et chefs d'industries sont d'accords sur un point : la $5 \mathrm{G}$, la nouvelle génération de technologie mobile, ne sera vraiment notable qu'à partir de 2023 au mieux, avec l'émergence de véritables cas d'usages dignes de ce nom. Cela n'empêche pas certains géants du numérique de ne pas attendre pour rêver tout haut de ce que sera la 6G, qui n'est encore qu'un sigle dénué de toute réalité tangible ».

Samsung dans le livre blanc ${ }^{13}$, prévoit que l'achèvement de la norme $6 \mathrm{G}$ et sa première date de commercialisation pourraient débuter à compter de 2028 au plus tôt, avant une commercialisation massive aux alentours de 2030. Et de prédire que cette nouvelle génération de technologie sans fil pourrait donner lieu à des débits de pointe théoriques de $1000 \mathrm{~Gb} / \mathrm{s}$ ainsi qu'à un temps de latence inférieur à 100 microsecondes $(\mu \mathrm{s})$, soit 50 fois le débit de données de pointe et un dixième de la latence de la $5 \mathrm{G}$. L'objectif derrière cette première expérimentation est de tester la communication entre un satellite et le sol terrestre, un moyen de réduire le temps de transmission entre l'espace et la Terre mais aussi d'améliorer la couverture Internet sur notre planète, notamment dans les zones blanches

\section{Conclusion}

Au-delà d'un débit toujours plus rapide, la 6G pourrait bien nous permettre d'accéder à des technologies jusqu'alors très peu développées. La 6G permettra par exemple aux smartphones dernière génération d'afficher des hologrammes volumétriques réels.

Entre Paris et Berlin, la « souveraineté technologique », qui suppose un savoir-faire propre et une certaine distance avec Washington, pose beaucoup moins de problèmes que l'épineuse question de la défense. En témoignent l'élargissement en novembre de GaiaX ${ }^{14}$, le « cloud européen », initiative franco-allemande, ou la coopération industrielle européenne sur certains secteurs comme les semiconducteurs ou les batteries.

Les fournisseurs européens Nokia et Ericsson n'ont pas été suffisamment soutenus, ils sont plus coûteux et moins avancés que Huawei, explique un observateur averti des milieux industriels allemands. Aujourd'hui, on a compris que la 5G est un élément central de la nouvelle phase industrielle qui s'amorce, où la donnée ${ }^{15}$ est au centre de la création de valeur.

\footnotetext{
${ }^{12}$ Pierre Benhamou : https://www.zdnet.fr/actualites/samsung-brosse-le-portrait-de-ce-que-pourrait-etre-la-6g-39906835.htm

${ }^{13}$ https://www.samsung.com/fr/business/smart-retail/livre-blanc/

${ }^{14}$ GAIA-X est un projet initié par l'Europe pour l'Europe. Son objectif est de développer des exigences communes pour une infrastructure de données européenne. " GAIA-X ; des représentants de la politique, des affaires et de la science de France et d'Allemagne, ainsi que d'autres partenaires européens, créent une proposition pour la prochaine génération d'une infrastructure de données pour l'Europe : un système sécurisé et fédéré qui répond aux plus hauts standards de souveraineté numérique tout en favorisant l'innovation. Ce projet est le berceau d'un écosystème numérique ouvert et transparent, où les données et les services peuvent être mis à disposition, rassemblés et partagés dans un environnement de confiance »

15 « Big Data, Open Data et Développement de données » Jean-Louis Monino, Soraya Sedkaoui Editeur ISTE version anglaise Editeur WILEY. 2016
}

"Contrôle des données : Défi majeur pour la société numérique » Jean-Louis Monino, Université de Montpellier, Volume 29 - Smart Innovation SET coordonné par Dimitri Uzunidis octobre 2020 France 
L'objectif est de leur permettre d'échanger rapidement de grandes quantités de données de façon sécurisée. L'industrie 4.0, qui repose sur la production et la transmission de données entre machines, est considérée comme l'avenir du made in Germany.

Selon Hapticmedia ${ }^{16}$ " l'un des plus grands défis auquel l'industrie 4.0 devra faire face est le bouleversement du marché du travail causé par l'automation, la robotique avancée et l'IA. Les ordinateurs et machines seront en mesure d'accomplir des tâches de plus en plus compliquées et, par conséquent, mettront en péril des millions d'emplois. Un expert dans le domaine de l'IA a même estimé que cette dernière pourrait remplacer jusqu'à $40 \%$ des emplois existants d'ici les 15 prochaines années ».

Un an à peine après le déploiement de la $5 \mathrm{G}$ dans le pays, la Chine vient d'annoncer avoir déployé un satellite $6 \mathrm{G}$ en orbite autour de la Terre. La course à l'espace est plus que jamais lancée. Cette technologie devrait être 100 fois plus rapide que la 5G. Le 6 novembre 2020, les chinois ont envoyé un satellite nouvelle génération dans l'espace dont l'objectif sera d'étudier les échanges de données à l'aide de réseau dont la fréquence atteint le térahertz, dans un milieu spatial. Pour comprendre la puissance de cette technologie, il faut la comparer avec la 5G. Les fréquences millimétriques utilisées avec la $5 \mathrm{G}$ varient de 30 à $300 \mathrm{GHz}$.

Le satellite doit également permettre de surveiller et de détecter les catastrophes naturelles comme les incendies de forêt, mais sera aussi utile pour superviser les ressources forestières, surveiller la conservation de l'eau.

La 6G n'en est encore qu'à ses débuts mais elle est prometteuse. Selon Samsung cette technologie pourrait être disponible dès 2028.

L'Europe se lance également dans le développement de la 6G. Concrètement, les réseaux $6 \mathrm{G}$ devront « comprendre » les besoins des utilisateurs pour mieux les anticiper. De quoi repousser encore plus les limites de l'interface humain-machine.

Début janvier 2021, le projet « Hexa-X $»^{17}$ dédié à la $6 \mathrm{G}$ est officiel financé par l'Union européenne. Le projet «Hexa-X ${ }^{18}$ est piloté principalement par Nokia et à son côté de Ericsson, une vingtaine d'entreprises dont Orange et Atos pour la France.

Stéphane Le Calme, de Développez.com, rappelle que " Nokia a été à l'avant-garde de la commercialisation de chaque génération de technologie sans fil, du premier appel GSM aux réseaux $5 \mathrm{G}$ en passant par les réseaux $4 \mathrm{G}$ ».

Peter Vetter, Head of Access Research Lab de chez Nokia «Les cas pratiques, le développement de technologies $6 \mathrm{G}$ fondamentales et la définition d'une nouvelle architecture pour une matrice intelligente qui intègre les activateurs technologiques $6 \mathrm{G}$ essentiels $»$.

\footnotetext{
${ }^{16}$ https://hapticmedia.fr/a-propos/

${ }^{17}$ Le projet « $6 \mathrm{G}$ Hexa-X » réuni ; des industriels, des acteurs de la chaîne de valeur complète des futures solutions de connectivité, des fournisseurs de réseaux, des opérateurs, des instituts de recherche et universités européens. Une masse d'experts pour mener un effort pour intégrer la recherche et le développement vers la 6G. le site : https://hexa-x.eu/about/

${ }^{18}$ Elsa Bembaron, Journaliste au Figaro, dans son article du 6 janvier 2021 « $6 \mathrm{G}$ : le départ à ne pas manquer » site : https://www.lefigaro.fr/secteur/high-tech/6g-le-depart-a-ne-pas-manquer-20210106
} 


\section{Webographie}

https://www.arcep.fr/nos-sujets/parlons-5g-toutes-vos-questions-sur-la-5g.html

https://www.francetvinfo.fr/internet/telephonie/5g/la-5g-dangereuse-inutile-ou-encore-polluante-on-a-examine-a-laloupe-les-arguments-des-elus-de-gauche_4106859.html

https://www.usinenouvelle.com/article/voici-1-un-des-cas-d-usage-les-plus-prometteurs-de-la-5g-et-il-ne-releve-pas-desmobiles.N1006859

https://www.publicsenat.fr/article/politique/deploiement-de-la-5g-le-gouvernement-ne-veut-pas-d-interferences-avec-les https://www.economie.gouv.fr/lancement-procedure-attribution-frequences-5g

https://vudailleurs.com/la-5g-est-un-formidable-outil-de-productivite-economique-et-ecologique-jacques-attali/ https://www.itu.int/web/pp-18/fr/backgrounder/5g-fifth-generation-of-mobile-technologies

https://www.anses.fr/fr/content/la-technologie-5g

https://www.anfr.fr/accueil/

https://fr.idate.org/note-enjeux-5g/

https://www.01net.com/actualites/pourquoi-la-5g-est-une-mauvaise-nouvelle-pour-1-environnement-1846716.html 